\title{
SINTOMAS DE ESTRESSE EM TRABALHADORAS DE ENFERMAGEM DE UMA UNIDADE DE PRONTO SOCORRO
}

\author{
Maycon Rogério SELEGHIMa, Mônica Augusta MOMBELLI ${ }^{\mathrm{b}}$, Magda Lúcia Félix de OLIVEIRAc,
} Maria Angélica Pagliarini WAIDMAN ${ }^{\mathrm{d}}$, Sonia Silva MARCON ${ }^{\mathrm{e}}$

\section{RESUMO}

Estudo transversal, realizado em um Pronto-Socorro, com o objetivo de identificar a associação de dados sociodemográficos, ocupacionais e econômicos em trabalhadores de enfermagem com a presença de sintomas de estresse. Os dados foram coletados em julho e agosto de 2009, junto a 33 sujeitos, utilizando um Inventário de Sintomas de Estresse para Adultos. A maioria das trabalhadoras apresentava sintomas de estresse nas fases de resistência e de quase exaustão, com presença de sintomas psicológicos e físicos. Maiores frequências foram encontradas em trabalhadoras mais jovens, casadas, com ensino superior completo, católicas, que não possuem outro emprego, trabalham no período vespertino ou noturno e, como técnicas de enfermagem, atuam no setor há mais de seis anos e na enfermagem há menos de dez anos. Conclui-se, pela necessidade de atuação junto a essas trabalhadoras com o intuito de prevenir novos casos, tratar os existentes, a fim de evitar a evolução para estágios mais graves.

Descritores: Esgotamento profissional. Estresse psicológico. Enfermagem em emergência. Condições de trabalho.

\section{RESUMEN}

Estudio transversal realizado en el Unidad de Urgencias con el objetivo de identificar la asociación de los datos sociodemográficos, laborales y económicos con la presencia de síntomas de estrés en trabajadores de enfermería. Los datos fueron recolectados en julio y agosto de 2009, junto a 33 sujetos, utilizando un Inventario de Síntomas de Estrés para Adultos. La mayoría de las trabajadoras presentaba síntomas de estrés en las fases de resistencia y de casi agotamiento, con presencia de síntomas psicológicos y físicos. Mayores frecuencias fueron encontradas en trabajadoras más jóvenes, casadas, con enseñanza superior completa, católicas, que no tienen otro empleo, trabajan en el período vespertino o nocturno y como técnicas de enfermería, actúan en el sector hace más de seis años y en la enfermería hace menos de 10 años. Se concluye por la necesidad de actuación junto a esas trabajadoras, para prevenir nuevos casos, tratar los existentes evitando la evolución para niveles más graves.

Descriptores: Agotamiento profesional. Estrés psicológico. Enfermería de urgencia. Condiciones de trabajo. Título: Síntomas de estrés en las trabajadoras de enfermería de una unidad de hospital de urgencias.

\section{ABSTRACT}

This is a sectional study carried out in an Emergency Room, with the purpose of identifying the association of socio-demographic, occupational and economic data in nursing workers presenting stress symptoms. Data was collected from 33 subjects, in July and August 2009, using an Inventory of Stress Symptoms for Adults. Results show that most of the workers presented stress symptoms at resistance and almost-exhaustion stages, with presence of psychological and physical symptoms. The highest occurrence was found in female younger workers that were married, graduated, catholic, without a second job, working as nursing technicians in the afternoon or at night, in the Emergency Room for more than six years, and in the Nursing field for less than ten years. It was concluded that something should be done to treat the existing cases of stress in order to avoid their evolution to more serious stages, and prevent new occurrences.

Descriptors: Professional burnoutl. Psychological stress. Emergency nursing. Working conditions.

Title: Stress symptoms in female nurses working in emergency rooms.

a Enfermeiro do Hospital Paraná. Mestre em Enfermagem.

b Psicóloga da Secretaria de Saúde de Matelândia. Mestre em Ciências da Saúde.

c Enfermeira. Doutora em Saúde Coletiva. Professora da Graduação e Pós-graduação em Enfermagem da Universidade Estadual de Maringá - UEM.

d Enfermeira. Doutora em Filosofia da Enfermagem. Professora da Graduação e Pós-graduação em Enfermagem da UEM.

e Enfermeira. Doutora em Filosofia da Enfermagem. Professora da Graduação e Pós-graduação em Enfermagem e da pós-graduação em Ciências da Saúde da UEM 


\section{INTRODUÇÃO}

Nos últimos anos, observa-se o aumento de pesquisas sobre o estresse, por seus efeitos na qualidade de vida dos indivíduos. O estresse na sociedade moderna pode ser explicado pelas transformações sociais que vêm desenhando o modo de vida e definindo novos padrões de saúde-doença, e exerce forte influência sobre a estrutura de trabalho ${ }^{(1)}$.

Quando o organismo é exposto a um esforço ou um estímulo percebido como ameaçador ao seu equilíbrio, seja ele um agente físico, químico, biológico ou psicossocial, o corpo se organizará para responder sob a forma de adaptação(2). Em 1956, foi proposto um modelo representativo do quadro de estresse, constituído de três fases: alarme, resistência e exaustão ${ }^{(3)}$.

$\mathrm{Na}$ fase de alarme, a pessoa reconhece o estímulo estressor e, como forma de enfrentamento, experimenta uma série de sensações, como sudorese nas mãos, taquipneia, taquicardia, e epigastralgia. Na fase de resistência, o organismo tenta restabelecer seu equilíbrio interno, reparando os danos causados na fase inicial. Se o agente ou estímulo estressor continuar, o organismo evolui à terceira fase, a de exaustão. Nesta fase, ressurgem os sinais e sintomas ocorridos na fase inicial e reações orgânicas com maior comprometimento físico e psíquico de adaptação, que se manifesta em forma de doenças ${ }^{(2-3)}$.

Posteriormente, foi identificada uma quarta fase, denominada de quase-exaustão, que ocorre quando a pessoa não consegue mais adaptar-se ou resistir aos estímulos estressores, ocasionando doenças pelo enfraquecimento do organismo ${ }^{(4)}$.

$\mathrm{O}$ estresse vinculado ao trabalho ou estresse ocupacional, refere-se à falta de capacidade do trabalhador de se (re)adaptar às demandas existentes no trabalho. Este tipo de estresse pode, ainda, referir-se ao conjunto de perturbações psicológicas e ao sofrimento psíquico associados às experiências de trabalho, cujas demandas ultrapassam as capacidades físicas ou psíquicas do sujeito para enfrentar as solicitações do ambiente profissional ${ }^{(5)}$. Estudar o estresse em trabalhadores de saúde tem sido importante porque, dependendo do grau do mesmo, aparecem doenças que levam ao absenteísmo, causando prejuízo para o trabalhador e a instituição empregadora ${ }^{(6)}$.

Estudo realizado em um hospital geral no Rio de Janeiro constatou que o terceiro motivo mais observado de licenças de saúde por períodos iguais ou superiores a 15 dias foram os transtornos mentais e comportamentais $^{(7)}$. As condições de trabalho vivenciadas pelos membros da equipe de enfermagem no ambiente hospitalar têm ocasionado vários problemas de saúde, acarretando prejuízos pessoais, sociais e econômicos ${ }^{(5)}$. Condições de trabalho inadequadas (jornadas extensas, ausência de períodos de descanso, plantões em finais de semana) e da ausência de participação nos processos de tomada de decisões podem interferir na saúde do trabalhador e também causar insatisfação profissional ${ }^{(5-6)}$.

Os trabalhadores de enfermagem, que atuam em unidades hospitalares de atendimento às urgências, vivenciam uma variedade de problemas relacionados às condições de trabalho que potencializam os fatores que favorecem o estresse: atendem uma demanda que ultrapassa a capacidade de intervenção dos serviços nas $24 \mathrm{~h}$ do dia, e as pessoas possuem agravos à saúde que requerem atendimento imediato ${ }^{(8-9)}$.

Exige-se dos trabalhadores de enfermagem que atuam nessas unidades: capacidade de agir sob tensão, alta habilidade psicomotora e aptidão, pois, geralmente, este ambiente é caracterizado por um número excessivo de pacientes, em extrema diversidade de condições clínicas, desde estados críticos até quadros estáveis, e entrada frequente de pacientes que necessitam de atendimento de urgência, sendo comum a escassez de recursos humanos e materiais, supervisão inadequada, e falta de valorização dos profissionais envolvidos ${ }^{(10)}$.

Estas características por si só podem ser desencadeadoras de estresse e a identificação de sua presença é relevante e necessária para cuidar da saúde dos trabalhadores da equipe de enfermagem que atuam nas unidades de pronto socorro (PS). Diante do exposto, o objetivo do presente estudo foi identificar a associação de dados sociodemográficos, ocupacionais e econômicos com a presença de sintomas de estresse em trabalhadores de enfermagem de uma unidade de PS da região Sul do Brasil.

\section{MÉTODOS}

Estudo transversal, realizado em uma unidade de PS de um hospital público de médio porte, considerado referência em atendimento às urgências para a região Noroeste do Estado do Paraná.

Essa unidade atende em regime de plantão permanente, com média diária de atenção a 150 pacientes; conta com 31 leitos para observação clínica de pacientes, os quais são utilizados também para internação de 
longa permanência pela falta de leitos para transferência inter-hospitalar. No período estudado, a unidade contava com 57 trabalhadores de enfermagem - 12 enfermeiros e 45 técnicos de enfermagem.

Foram convidados a participar da pesquisa, por meio de abordagem direta, todos os trabalhadores de enfermagem que atuavam na sala de estabilização e reanimação, e na unidade de cuidados intensivos do PS, totalizando dez enfermeiros e 29 técnicos de enfermagem.

A sala de estabilização e reanimação e a unidade de cuidados intensivos são destinadas ao atendimento de pacientes críticos; na primeira são instituídas as medidas iniciais de suporte de vida, e a segunda funciona como uma sala de cuidados intensivos, onde pacientes em estado crítico aguardam transferência para unidades de terapia intensiva ${ }^{(10)}$.

Os dados foram coletados nos meses de julho e agosto de 2009, por meio de dois instrumentos autoaplicáveis entregues aos trabalhadores no momento da abordagem e devolvidos no prazo máximo de 30 dias, após várias solicitações A pesquisa contou com 33 informantes, visto que seis não devolveram os questionários preenchidos.

Os instrumentos foram um formulário semiestuturado com 11 itens, elaborado pelos autores e destinado à coleta de dados sociodemográficos, ocupacionais e econômicos (categoria profissional, turno de trabalho, tempo no setor, outro emprego, tempo de profissão, e renda); e o Inventário de Sintomas de Stress para Adultos (ISSA) ${ }^{(11)}$, que permite identificar, a partir da sintomatologia relatada pelo indivíduo, a presença de estresse, o tipo de sintoma presente - físico, psicológico ou físico-psicológico, e a fase do estresse - alarme, resistência, quase-exaustão e exaustão ${ }^{(11)}$.

O ISSA é composto por três quadros que se referem às quatro fases do estresse. $\mathrm{O}$ primeiro quadro (fase de alarme) é composto por 12 sintomas físicos e três psicológicos; o respondente assinala os sintomas experimentados nas últimas $24 \mathrm{~h}$. O segundo quadro (fase de resistência) é composto de dez sintomas físicos e cinco psicológicos; sendo assinalado os sintomas experimentados na última semana. No terceiro quadro (fase de exaustão), composto por 12 sintomas físicos e 11 psicológicos, o respondente assinala os sintomas experimentados no último mês. A terceira fase (quase-exaustão) é diagnosticada com base na frequência dos itens assinalados na fase de resistência ${ }^{(11)}$.
Os dados da aplicação dos instrumentos foram compilados e comparados no programa computacional Excel Access ${ }^{\circledR}$ 2003, seguindo as etapas do Manual do Inventário de Sintomas de Estresse para Adultos ${ }^{(12)}$ para a interpretação do ISSA. Para identificação da associação dos dados sociodemográficos, ocupacionais e econômicos com a presença de sintomas de estresse, foi realizada a regressão logística no programa computacional Statistical Analisys System ${ }^{\circledR} 9.1(\mathrm{p}<0,05)$.

O estudo foi aprovado pelo Comitê Permanente de Ética em Pesquisas com Seres Humanos da Universidade Estadual de Maringá (Parecer $n^{\circ}$ 319/2009). Todos os participantes assinaram o Termo de Consentimento Livre e Esclarecido.

\section{RESULTADOS}

Os 33 sujeitos eram do sexo feminino, com idade de 24 a 53 anos. Grande parte era casada (45,6\%), possuía filhos $(69,7 \%)$ e professava a fé católica (57,5\%). Quanto à escolaridade, 21 (63,6\%) informaram o curso superior completo, sendo que sete (33,3\%) já cursaram especialização ou mestrado. Em relação às características ocupacionais e econômicas, a maior parte era técnica de enfermagem $(75,7 \%) ; 22(66,6 \%)$ trabalhavam no período noturno, seis $(18,2)$ no matutino e cinco $(15,2 \%)$ no vespertino, e 69,7\% trabalhava no PS há menos de cincos anos e 84,7\% não possuía outro emprego.

Dez entrevistadas (30,3\%) trabalhavam entre quatro a dez anos na Enfermagem, 12 (36,4\%) entre 11 a 20 anos, e 11 (33,3\%) há mais de 20 anos. A renda mensal se situava entre três a cinco salários mínimos (60,6\%) e a carga horária de trabalho de todas as entrevistadas era de $36 \mathrm{~h}$ semanais, sendo que a totalidade delas realizava até $60 \mathrm{~h}$ extras por mês.

A aplicação do ISSL identificou que 23 (70,0\%) entrevistadas apresentavam sintomas de estresse nas fases de resistência e de quase-exaustão. Destas, quatro eram enfermeiras, representando 50,0\% do total de enfermeiras entrevistadas, e 19 eram técnicas de enfermagem, representando 76,0\% das trabalhadoras dessa categoria profissional.

Todas as enfermeiras que apresentaram estresse encontravam-se na fase de resistência, e das técnicas de enfermagem, 17 (68,0\%) se encontravam na fase de resistência e duas $(8,0 \%)$ na fase de quase-exaustão. Nenhuma entrevistada encontrava-se na fase de alarme ou exaustão (Tabela 1). 
Os resultados indicaram que, dentre aquelas que apresentaram sintomas de estresse, houve predomínio dos sintomas psicológicos (56,0\%), seguido dos sintomas físicos (35,0\%) e físico-psicológicos $(9,0 \%)$.

Independentemente das fases do estresse, os sintomas físicos mais relatados pelas enfermeiras foram o problema de memória e o cansaço constante $(100 \%$ cada), e pelas técnicas de enfermagem, a sensação de desgaste físico constante $(84,2 \%)$. Quanto aos sintomas psicológicos, verificou-se que a sensibilidade emotiva excessiva e a irritabilidade excessiva foram os mais relatados nas duas categorias profissionais (Tabela 2 ).

A associação dos dados sociodemográficos com a presença de sintomas de estresse pode ser observado na Tabela 3. Verificou-se maior frequência

Tabela 1 - Distribuição das trabalhadoras de enfermagem segundo as fases do estresse e categoria profissional. Maringá, PR, 2009.

\begin{tabular}{lcccc}
\hline \multirow{2}{*}{ Fases do estresse } & \multicolumn{2}{c}{ Enfermeira } & \multicolumn{2}{c}{ Técnica de enfermagem } \\
\cline { 2 - 5 } & $\mathbf{n}$ & $\mathbf{\%}$ & $\mathbf{n}$ & $\mathbf{\%}$ \\
\hline Sem estresse & 04 & 50,0 & 06 & 24,0 \\
Resistência & 04 & 50,0 & 17 & 68,0 \\
Quase-exaustão & -- & -- & 02 & 8,0 \\
\hline Total & 08 & 100,0 & 25 & 100,0 \\
\hline
\end{tabular}

Tabela 2 - Distribuição das trabalhadoras de enfermagem, segundo o tipo de sintoma e a categoria profissional. Maringá, PR, 2009.

\begin{tabular}{lcccc}
\hline \multicolumn{1}{c}{ Tipo de sintoma* } & \multicolumn{2}{c}{$\begin{array}{c}\text { Enfermeira } \\
(\mathbf{n = 4})\end{array}$} & \multicolumn{2}{c}{$\begin{array}{c}\text { Técnica de enfermagem } \\
(\mathbf{n = 1 9 )}\end{array}$} \\
\cline { 2 - 5 } Físicos & $\mathbf{n}$ & $\mathbf{0}$ & $\mathbf{n}$ & $\mathbf{\%}$ \\
Problema de memória & & & & \\
Mal-estar generalizado sem causa específica & 04 & 100,0 & 12 & 63,1 \\
Formigamento das extremidades & 01 & 25,0 & 08 & 42,1 \\
Sensação de desgaste físico constante & 01 & 25,0 & 01 & 5,2 \\
Mudança de apetite & 03 & 75,0 & 16 & 84,2 \\
Aparecimento de problemas dermatológicos & 03 & 75,0 & 08 & 42,1 \\
Hipertensão arterial & -- & -- & 03 & 15,7 \\
Cansaço constante & -- & -- & 02 & 10,5 \\
Aparecimento de úlcera & 04 & 100,0 & 13 & 68,4 \\
Tontura/sensação de estar flutuando & -- & -- & 01 & 5,2 \\
Psicológicos & -- & -- & 02 & 10,5 \\
Sensibilidade emotiva excessiva & & & & \\
Dúvida quanto a si própria & 03 & 75,0 & 13 & 68,4 \\
Pensar constantemente em um só assunto & -- & -- & 06 & 31,5 \\
Irritabilidade excessiva & 02 & 50,0 & 08 & 42,1 \\
Diminuição da libido & 03 & 75,0 & 12 & 63,1 \\
\hline
\end{tabular}

* Sintomas das fases de resistência e de quase-exaustão. 
Tabela 3 - Distribuição logística da associação entre presença de sintomas de estresse e dados sociodemográficos. Maringá, PR, 2009.

\begin{tabular}{|c|c|c|c|c|c|c|}
\hline \multirow{3}{*}{ Variáveis } & \multirow{3}{*}{$(\mathrm{n}=33)$} & \multicolumn{4}{|c|}{ Presença de sintomas de estresse } & \multirow{3}{*}{$\mathbf{P}^{*}$} \\
\hline & & \multicolumn{2}{|c|}{$\operatorname{SIM}(n=23)$} & \multicolumn{2}{|c|}{ NÃO $(n=10)$} & \\
\hline & & $\mathbf{n}$ & $\%$ & $\mathbf{n}$ & $\%$ & \\
\hline Faixa etária (anos) & & & & & & 0,1516 \\
\hline $24-39$ & 17 & 13 & 76,4 & 04 & 23,6 & \\
\hline $40-53$ & 16 & 10 & 62,5 & 06 & 37,5 & \\
\hline Escolaridade & & & & & & 0,8716 \\
\hline Ensino Médio & 12 & 08 & 66,6 & 04 & 33,4 & \\
\hline Superior completo & 21 & 15 & 71,4 & 06 & 28,6 & \\
\hline Estado civil & & & & & & 0,2421 \\
\hline Solteira & 09 & 05 & 55,5 & 04 & 44,5 & \\
\hline Casada & 15 & 13 & 86,6 & $\mathrm{O} 2$ & 13,4 & \\
\hline Divorciada/Viúva & 09 & 05 & 55,5 & 04 & 44,5 & \\
\hline Filhos & & & & & & 0,3292 \\
\hline $\operatorname{Sim}$ & 23 & 16 & 69,5 & 07 & 30,5 & \\
\hline Não & 10 & 07 & 70,0 & 03 & 30,0 & \\
\hline Religião & & & & & & 0,7372 \\
\hline Católica & 19 & 15 & 78,9 & O4 & 21,1 & \\
\hline Evangélica & 09 & 05 & 55,5 & 04 & 44,5 & \\
\hline Outra & 05 & 03 & 60,0 & $\mathrm{O} 2$ & 40,0 & \\
\hline
\end{tabular}

Nota: $*$ p-valor.

de sintomas de estresse entre trabalhadoras na faixa etária de 24 a 39 anos $(13-76,4 \%)$, com ensino superior completo $(15-71,4 \%)$, casadas $(13-86,6 \%)$ e católicas (15-78,9\%). Porém, a análise estatística não mostrou associação significativa entre as variáveis analisadas.

A associação dos dados ocupacionais e econômicos com a presença de sintomas de estresse pode ser observada na Tabela 4. Maior frequência de sintomas de estresse foi verificado em técnicas de enfermagem $(19-76,0)$, em trabalhadoras do turno de vespertino $(4-80 \%)$ ou noturno $(16$ $-72,7 \%$ ), que atuam no PS por tempo igual ou maior que seis anos $(8-80,0 \%)$ e na enfermagem entre quatro a dez anos $(8-80 \%)$, que possuem um único vínculo empregatício $(19-67,8 \%)$ e renda mensal entre três a cinco salários mínimos $(16-80,0 \%)$. A análise estatística também não apontou associação entre estas variáveis e a presença de sintomas de estresse.

\section{DISCUSSÃO}

O estresse é um agravo multifatorial e os traços individuais são determinantes para a sua ocorrência, caracterizada pelo desgaste do indivíduo na luta constante pelo sucesso e para satisfazer os ideais de excelência determinados pela sociedade e pelo trabalho ${ }^{(3-4)}$.

O ISSA identificou que a maioria das trabalhadoras apresentava sintomas de estresse, corroborando dados da literatura que têm identificado altos índices de estresse na equipe de enfermagem, principalmente no ambiente hospitalar ${ }^{(1,5-6,6,13)}$. Profissionais de enfermagem precisam ter tempo para descansos e pausas, pois, se não houver períodos adequados para a recuperação do estresse fisiológico e mental provocado pela função, apresentam potencial para o adoecimento. Isto é particularmente afetado quando, por necessidade pessoal ou da instituição, o profissional realiza muitas horas extras 
Tabela 4 - Distribuição logística da associação entre presença de sintomas de estresse e dados ocupacionais e econômicos. Maringá, PR, 2009.

\begin{tabular}{|c|c|c|c|c|c|c|}
\hline \multirow{3}{*}{ Variáveis } & \multirow{3}{*}{$(\mathrm{n}=33)$} & \multicolumn{4}{|c|}{ Presença de sintomas de estresse } & \multirow{3}{*}{$\mathbf{P}^{*}$} \\
\hline & & \multicolumn{2}{|c|}{$\operatorname{SIM}(n=23)$} & \multicolumn{2}{|c|}{ NÃO $(n=10)$} & \\
\hline & & $\mathrm{n}$ & $\%$ & $\mathrm{n}$ & $\%$ & \\
\hline Categoria profissional & & & & & & 0,6419 \\
\hline Enfermeira & 08 & 04 & 50,0 & 04 & 50,0 & \\
\hline Técnica de enfermagem & 25 & 19 & 76,0 & 06 & 24,0 & \\
\hline Turno de trabalho & & & & & & 0,5138 \\
\hline Matutino & 06 & 03 & 50,0 & 03 & 50,0 & \\
\hline Vespertino & 05 & 04 & 80,0 & 01 & 20,0 & \\
\hline Noturno & 22 & 16 & 72,7 & 06 & 27,3 & \\
\hline Tempo no setor & & & & & & 0,1996 \\
\hline$<6$ anos & 23 & 15 & 65,2 & 08 & 34,8 & \\
\hline$\geq 6$ anos & 10 & 08 & 80,0 & 02 & 20,0 & \\
\hline Outro emprego & & & & & & 0,9269 \\
\hline Não & 28 & 19 & 67,8 & 09 & 32,2 & \\
\hline $\operatorname{Sim}$ & 05 & 03 & 60,0 & $\mathrm{O} 2$ & 40,0 & \\
\hline Tempo na enfermagem & & & & & & 0,6603 \\
\hline $4-10$ anos & 10 & 08 & 80,0 & 02 & 20,0 & \\
\hline $11-20$ anos & 12 & 08 & 66,6 & 04 & 33,4 & \\
\hline$>20$ anos & 11 & 07 & 63,6 & 04 & 36,4 & \\
\hline Renda mensal $(\mathrm{SM})^{* *}$ & & & & & & 0,5616 \\
\hline $3-5$ & 20 & 16 & 80,0 & 04 & 20,0 & \\
\hline 6 ou mais & 13 & 07 & 53,8 & 06 & 46,2 & \\
\hline
\end{tabular}

Nota: * p-valor; **SM: salário mínimo vigente no período de realização da pesquisa $(\mathrm{R} \$ 465,00)$.

ou então mantém dupla ou até tripla jornada de trabalho, comprometendo o período de recuperação ${ }^{(7)}$.

Além do desenvolvimento de atividades com pacientes críticos e em risco eminente de morte, as características pessoais das trabalhadoras apresentaram vários fatores considerados de risco para o desenvolvimento de estresse, como o sexo feminino, ciclo vital adulto, estado civil casado, ter filhos, duplo vínculo e trabalho no período noturno ${ }^{(1,6,13-14)}$.

Estudo realizado com profissionais de um pronto atendimento relatou que estes profissionais percebem mais os sintomas físicos do estresse que os psíquicos, associados à resistência dos profissionais, provocando negação dos sintomas psíquicos ${ }^{(6)}$. Este achado corrobora o presente estudo, em que foi observado maior destaque aos sintomas físicos do que psíquicos.
Uma vez que a população em estudo foi constituída pelo sexo feminino, a associação da presença de sintomas de estresse entre os sexos ficou limitada. Contudo, o estresse em mulheres ocorre com maior frequência pela sobrecarga de tarefas características do mundo feminino, pela dupla ou tripla jornada de trabalho ${ }^{(15)}$.

No estudo, o fator "sexo feminino" alia-se à idade adulta, com responsabilidades e exigências geradas por esta fase da vida, como a necessidade de autorrealização pessoal e profissional e o provimento de recursos financeiros para o sustento da família, bem como ao estado civil e à presença e responsabilidade com filhos como indicadores de sobrecarga, pelo acúmulo das funções intrafamiliares ${ }^{(13)}$.

Apesar de os sintomas de estresse serem mais frequentes entre as trabalhadoras do turno 
vespertino, a atuação no período noturno requer maior atenção, visto que pode causar uma série de distúrbios fisiológicos e psicossociais, pelas mudanças dos ritmos biológicos e pela dessincronização familiar e social da vida do trabalhador ${ }^{(15)}$.

O contínuo atraso do sono pelos horários de trabalho pode levar à insônia e, consequentemente ao estresse. Os trabalhadores em turnos ou em trabalho noturno, geralmente, possuem sono de má qualidade no período diurno, em decorrência dos conflitos sociais - 'coisas que fazemos de dia e coisas que fazemos de noite’ - e do excesso de ruído diurno ${ }^{(15)}$.

Destaca-se que as entrevistadas que trabalhavam no período noturno apresentaram frequência elevada de sintomas de estresse. O descompasso da convivência social em relação aos horários de trabalho pode levar ao isolamento social, tendo repercussões com início na família, estendendo-se a outros segmentos sociais. Além disso, após alguns anos nesta forma de trabalho, podem surgir manifestações crônicas como desordens do sono, doenças cardiovasculares e gastrintestinais, absenteísmo e divórcios ${ }^{(15)}$.

Pouco tempo de trabalho na enfermagem foi considerado fator de risco para o surgimento de estresse, pois as expectativas em relação ao desempenho das atividades são gradativamente afetadas pelas condições inadequadas de trabalho, levando à insatisfação profissional e ao desequilíbrio entre as exigências do trabalho e a capacidade para atendê-las ${ }^{(13,16)}$.

A ocorrência de sintomas entre as trabalhadoras que não possuíam outro emprego, pode ser justificada pela presença de elementos estressores no ambiente de trabalho, como o número excessivo de pacientes atendidos. Porém, o número excessivo de horas extras mensais, conformando o ciclo absenteísmo versus horas extras no PS, é fator determinante para o aumento da carga horária de trabalho em um setor desgastante e para a ocorrência do estresse ${ }^{(13)}$.

Com relação à religião, estudos apontam que a espiritualidade se mostra uma poderosa aliada no confronto com o estresse ${ }^{(17)}$. No entanto, a maioria das entrevistadas informou apenas vínculo à fé católica ou evangélica, de forma sistemática ou não, ficando a avaliação da associação prejudicada.

Quanto às fases do estresse, identificou-se que a maioria das entrevistadas encontrava-se na fase de resistência, revelando um desgaste acumulado ao longo do tempo. Esta fase é caracterizada por uma tentativa de recuperação do organismo após o desequilíbrio sofrido na fase anterior. Nesse momento, ocorre gasto de energia que pode ocasionar cansaço excessivo, problemas de memória e dúvidas quanto a si próprio, trazendo prejuízos à saúde e à qualidade da assistência oferecida ${ }^{(3)}$.

A presença de técnicas de enfermagem na fase de quase-exaustão indica agravamento do estresse. A cronicidade advinda da falta de resolutividade das fases iniciais do estresse faz com que a tensão e a resistência física e emocional excedam o limite gerenciável, trazendo prejuízos individuais e organizacionais ${ }^{(8)}$.

Quanto ao tipo de sintomas identificados naquelas que apresentaram estresse, houve predomínio dos sintomas psicológicos, corroborando outros estudos com profissionais da saúde, que apontam a prevalência de sintomas psicológicos em relação aos outros tipos de sintomas ${ }^{(18)}$. Maior prevalência de estresse em determinada área significa que a pessoa é mais vulnerável nessa área. Assim, sabendo qual a vulnerabilidade do indivíduo, medidas preventivas ou tratamentos específicos podem ser formulados, levando em consideração a maior predisposição a apresentar sintomas de uma natureza ou outra ${ }^{(12)}$.

Considerando que a maioria das trabalhadoras era técnica de enfermagem, a ocorrência de sintomas de estresse nessa categoria parece acontecer pela influência das atividades por elas desenvolvidas. No PS investigado, o técnico de enfermagem era o responsável pela assistência direta ao paciente, e o enfermeiro desenvolve atividades assistenciais, administrativas e de supervisão, que podem atuar como fator protetor para a autoestima profissional.

Outro fator a ser considerado para a manifestação do estresse é o ganho salarial, que mesmo elevado na população estudada, pode estar associado, de alguma forma, ao reconhecimento que as profissionais esperam pelo trabalho realizado no PS, corroborando estudos da área de Enfermagem ${ }^{(19)}$.

Dentre as consequências para a vida dos indivíduos com sintomas de estresse, podemos citar o comprometimento de quatro áreas principais: social, familiar/afetivo, de saúde e profissional. O individuo pode ter tendência ao isolamento e a conflitos interpessoais, as reações do estresse do indivíduo podem repercutir para todos os membros da família, uma variedade de doenças pode ser desencadeada pelo estresse excessivo, pelas alterações psicofisiológicas que ocorrem e pelas predisposições individuais, e observa-se absenteísmo, atrasos, queda da produtividade e problemas de relacionamento ${ }^{(20)}$. 


\section{CONCLUSÕES}

Com a aplicação do ISSA, verificou-se que a maioria das trabalhadoras de enfermagem investigadas apresentava sintomas de estresse. Houve, proporcionalmente, maior ocorrência de sintomas de estresse entre técnicas de enfermagem, visto que essas são responsáveis por todos os casos de estresse na fase de quase-exaustão, indicando que as atividades desenvolvidas por essa categoria profissional parecem contribuir para o surgimento de estresse, o que exigiria a realização de outros estudos para confirmação de tal suposição.

Encontraram-se possíveis fatores de risco para a ocorrência de estresse em trabalhadoras de enfermagem, como, por exemplo, encontrar-se na faixa etária de 24 a 39 anos, ser casada, ter filhos e trabalhar no período vespertino ou noturno, dentre outros.

A ausência de trabalhadoras com sintomas de estresse na fase de alarme ou exaustão indica que a situação ainda não é tão grave e que, portanto existe a possibilidade de atuação preventiva. Nestes casos, as próprias trabalhadoras devem ser convidadas para opinarem sobre os recursos/estratégias de enfretamento do estresse a serem adotadas coletiva e individualmente no ambiente de trabalho.

Vale destacar que o fato da população em estudo ser constituída em sua totalidade por trabalhadoras pode configurar limitação do estudo. No entanto, isto não prejudicou a discussão dos resultados encontrados.

Por fim, considera-se a temática abordada no estudo de grande importância para a Enfermagem como área de conhecimento, e mais especificamente a Enfermagem do Trabalho e Gerenciamento de Pessoas, à medida que fornece subsídios que possibilitam o desenvolvimento de ações mais efetivas de proteção à saúde dos trabalhadores que atuam em unidade de PS, e de forma mais específica, junto aqueles que apresentam características que os tornam mais vulneráveis ao estresse.

\section{REFERÊNCIAS}

1 Cavalheiro AM, Moura Junior DF, Lopes AC. Estresse de enfermeiros com atuação em unidade de terapia intensiva. Rev Latino-Am. Enfermagem. 2008;16(1):29-35.

2 Meneghini F, Paz AA, Lautert L. Fatores ocupacionais associados aos componentes da síndrome de
Burnout em trabalhadores de enfermagem. Texto Contexto Enferm. 2001; 20(2): 225-33

3 Selye HA. The stress of life. New York: MacGraw-Hill; 1956.

4 Lipp MEN. O modelo quadrifásico do stress. In: Lipp MEN, organizador. Mecanismos neuropsicofisiológicos do stress: teoria e aplicações clínicas. São Paulo: Casa do Psicólogo; 2003. p.17-21.

5 Schmidt DRC, Dantas RAS, Marziale MHP, Laus AM. Estresse ocupacional entre profissionais de enfermagem do bloco cirúrgico. Texto Contexto Enferm. 2009; 18(2):330-7.

6 Farias SMC, Teixeira OLC, Moreira W, Oliveira MAF, Pereira MO. Caracterização dos sintomas físicos de estresse na equipe de pronto atendimento. Rev Esc Enferm USP 2011; 45(3):722-9.

7 Carvalho LSF, Matos RCS, Souza NVDO, Ferreira REDS. Motivos de afastamento por licença de saúde dos trabalhadores de enfermagem. Cienc Cuid Saude. 2010; 9(1):60-6

8 Sá MC, Carreteiro TC, Fernandes MIA. Limites do cuidado: representações e processos inconscientes sobre a população na porta de entrada de um hospital de emergência. Cad Saude Publica. 2008; 24(6):1334-43.

9 Ministério da Saúde (BR). Portaria 2048/GM, de 5 de novembro de 2002. Brasília (DF); 2002.

10 Souza RB, Silva MJP, Nori A. Pronto-Socorro: uma visão sobre a interação entre profissionais de enfermagem e pacientes. Rev Gaúcha Enferm. 2007; 28(2):242-49.

11 Lipp MEN. Inventário de sintomas de estresse de Lipp. São Paulo: Casa do Psicólogo; 2000.

12 Lipp, MEN. Manual do inventário de sintomas de estresse para adultos de Lipp (ISSL). São Paulo: Casa do Psicólogo; 1998.

13 Panizzon C, Luz AMH, Fensterseifer LM. Estresse da equipe de enfermagem de emergência clínica. Rev Gaúcha Enferm. 2008; 29(3):391-399.

14 Hanzelmann, RS, Passos JP. Imagens e representações da enfermagem acerca do estresse e sua influência na atividade laboral. Rev Esc Enferm USP. 2010; 44(3):694-70 1 
15 Rosa PLFS, Fisher FM, Borges FNS, Soares NS, Rotenberg L, et al. Percepção da duração do sono e da fadiga entre trabalhadores de enfermagem. Rev Enferm UERJ. 2007;15(1):100-6.

16 Linch GFC, Guido LA. Estresse de enfermeiros em unidade de hemodinâmica no Rio Grande do Sul, Brasil. Rev Gaúcha Enferm. 2011;32(1):63-71

17 Panzini RG, Bandeira DR. Escala de Coping Religioso-Espiritual (Escala CRE): elaboração e validação de construto. Psicol Estud. 2005; 10(3);507-516.
18 Camelo SHH, Angerami ELS. Sintomas de estresse nos trabalhadores atuantes em cinco núcleos de saúde da família. Rev Latino-Am. Enferm. 2004; 12(1):14-21.

19 Batista AAV, Vieira MJ, Cardoso NCS, Carvalho GRP. Fatores de motivação e insatisfação no trabalho do enfermeiro. Rev Esc Enferm. USP. 2005; 39 (1);85-91.

20 Malagris NL, Fiorito CCA. Avaliação do nível de estresse de técnicos da área de saúde. Estud Psicol. 2006; 23(4) 391-398.

\section{Endereço do autor / Dirección del autor / Author's address}

Maycon Rogério Seleghim

Rua Osvaldo Cruz, 340, ap. 309, zona 07

87020-200, Maringá, PR

Telefone: (44) 8801-9447

E-mail:mseleghim@yahoo.com.br
Recebido em: 29.10.10

Aprovado em: 31.01.2012 